\title{
Causal sets and the deep structure of spacetime
}

\author{
Fay Dowker \\ Blackett Laboratory, Imperial College, London, SW7 2AZ, UK.
}

February 7, 2008

\begin{abstract}
The causal set approach to quantum gravity embodies the concepts of causality and discreteness. This article explores some foundational and conceptual issues within causal set theory.
\end{abstract}

\section{Introduction}

The problem of quantum gravity in its narrow sense is the problem of finding a theory that incorporates both Quantum Mechanics and General Relativity. A broader vision is that a theory of quantum gravity would restore to physics a unified framework, a framework in which there is no fundamental division in principle between observer and observed nor between matter and spacetime. One reading of Einstein's writings on Quantum Mechanics and Unification is that he viewed success in the broad quest as a prerequisite to a solution of the narrow problem.

Every approach to quantum gravity in this broad conception must embody answers to two fundamental questions: "What is quantum mechanics?" and "What is the deep structure of spacetime?" This article will touch on the former question and focus on the latter and the answer to it provided by the approach known as causal set theory which marries the two concepts of discreteness (or atomicity) and causality.

The view that causality is a more fundamental organising principle, even than space and time, is an ancient tradition of thought. Corresponding to this view, momentary events have a better claim to be basic than objects extended in time like particles, the latter being understood as persistent patterns of events rather than enduring "substances". Within Relativity, the recognition that almost all of the geometrical properties of Minkowski space could be reduced to order theoretic relationships among point events came very early. [1]

The concept of atomicity also has a long history as do philosophical challenges to the antithetical notion of a physical continuum. Of course Quantum Mechanics itself is named for the discreteness of atomic and subatomic phenomena. In more recent times, the increasing importance of computing with its discrete algorithms and digital processing has had a pervasive influence on intellectual culture. Whatever 
the roots of the concept, it is certainly now the case that many workers believe that a fundamentally discrete structure to reality is required to reconcile spacetime with the quantum. Einstein himself adumbrated this view and it is impossible to resist the temptation of quoting him here:

"But you have correctly grasped the drawback that the continuum brings. If the molecular view of matter is the correct (appropriate) one, i.e., if a part of the universe is to be represented by a finite number of moving points, then the continuum of the present theory contains too great a manifold of possibilities. I also believe that this too great is responsible for the fact that our present means of description miscarry with the quantum theory. The problem seems to me how one can formulate statements about a discontinuum without calling upon a continuum (space-time) as an aid; the latter should be banned from the theory as a supplementary construction not justified by the essence of the problem, which corresponds to nothing "real". But we still lack the mathematical structure unfortunately. How much have I already plagued myself in this way!"

A. Einstein in a letter to Walter Dällenbach, November 1916, translated and cited by John Stachel. [2]

Causal set theory [3, 4, 5, 6, 7, arises by combining discreteness and causality to create a substance that can be the basis of a theory of quantum gravity. Spacetime is thereby replaced by a vast assembly of discrete "elements" organised by means of "relations" between them into a "partially ordered set" or "poset" for short. None of the continuum attributes of spacetime, neither metric, topology nor differentiable structure, are retained, but emerge it is hoped as approximate concepts at macroscopic scales.

Amongst current approaches, causal set theory can claim to lie at the extreme end of the granularity scale: it is maximally discrete. No continuum concept is required to specify the underlying reality which is purely combinatorial. The elements have no internal structure; they are the fundamental units of reality. All one can do is count: count elements, count relations, count certain patterns of elements and relations. The slogan might be coined, "Real numbers are not real in a causal set."

The hypothesis that the deep structure of spacetime is a discrete poset characterises causal set theory at the kinematical level; that is, it is a proposition about what substance is the subject of the theory. However, kinematics needs to be completed by dynamics, or rules about how the substance behaves, if one is to have a complete theory. In this article I will explore some foundational issues within these two categories in a non-technical way. I have not attempted to give anything approaching a review of causal set theory. Reference [8], by Rafael Sorkin who has done more than anyone to further the causal set approach, gives more details of current developments. 


\section{Kinematics}

\subsection{The causal set}

Mathematically, a causal set is defined to be a locally finite partially ordered set, or in other words a set $C$ together with a relation $\prec$, called "precedes", which satisfy the following axioms:

(1) if $x \prec y$ and $y \prec z$ then $x \prec z, \forall x, y, z \in C$ (transitivity);

(2) if $x \prec y$ and $y \prec x$ then $x=y \forall x, y \in C$ (non-circularity);

(3) for any pair of fixed elements $x$ and $z$ of $C$, the set $\{y \mid x \prec y \prec z\}$ of elements lying between $x$ and $z$ is finite.

Of these axioms, the first two say that $C$ is a partially ordered set or poset and the third expresses local finiteness. The idea is that in the deep quantum regime of very small distances, spacetime is no longer described by a tensor field, the metric, on a differentiable manifold, but by a causal set. The discrete elements of the causal set are related to each other only by the partial ordering that corresponds to a microscopic notion of before and after, and the continuum notions of length and time arise only as approximations at large scales.

The richness of the structure of partial orders is reflected in the many different sets of terminologies used by mathematicians and physicists who study them. One of the most useful and suggestive for the purposes of quantum gravity is the "genealogical" jargon whereby one thinks of a causal set as a family tree. An element $x$ is an ancestor of an element $y$ if $x \prec y$, and $y$ is then a descendant of $x$.

To arrive at this structure as the kinematical basis for quantum gravity, one can start by conjecturing discreteness and see how this leads to the addition of causal structure, or vice versa. The scientific arguments for the two interweave each other and it's an artificial choice. Indeed, it is the fact that the two concepts "complete" each other in the context of a proposed substructure for spacetime that is one of the strongest motivations for the causal set programme. For the purposes of the current exposition let's choose to begin by postulating a discrete spacetime.

\subsection{An analogy: discrete matter}

In quantum gravity, we know what the continuum description of spacetime is at macroscopic scales - it is a Lorentzian manifold - and are trying to discover the discrete substratum. It is useful to imagine ourselves in the analogous situation for a simple physical system. Consider a quantity of a material substance in a box in flat space for which we have a continuum description - a mass density, say - but which we suspect is fundamentally discrete. The question is, "What could the discrete state be that gives rise to this continuum approximation?" and a good first response is to try to discretise the continuum. We then try to give the discrete object so created "a life of its own" as an entity independent of the continuum from which it was derived. We then ask whether we can believe in this discrete object as fundamental 
and whether and how we can recover from it a continuum approximation which may not be exactly the original continuum from which we started but which must be "close" to it.

In the case of the material substance, let us postulate that it is made of identical atoms. The varying mass density then can only be due to differing number densities of atoms in space. We discretise by somehow distributing the atoms in the box so that the number of atoms in each sufficiently large region is approximately proportional to the mass density integrated over that region. We may or may not have reasons to suspect what the atomic mass actually is, but in any case it must be small enough so that the spacing between the atoms is much smaller than the scales on which the density varies.

Each method of discretising will produce distributions of atoms that have different properties and which type is more favourable as a fundamental state depends on which features of the continuum theory we wish to preserve and how fruitful each turns out to be when we come to propose dynamics for the discrete structure itself. Suppose for example that the continuum theory of the material is invariant under Euclidean transformations (rotations and translations) at least locally in small enough regions over which the mass density is approximately constant and ignoring edge effects. There are ways to discretise which do not respect the invariance under Euclidean transformations. For example, we can divide the box into cubes small enough so the density is approximately constant in each. In each cube, place an atom at every vertex of a Cartesian lattice with lattice spacing chosen inversely proportional to the mass density in the cube. We can produce in this way an atomic state from which we can recover approximately the correct continuum mass density but it is not invariant under the Euclidean group - there are preferred directions - and if a fully fledged fundamental discrete theory is based on such lattice-like atomic states, this will show up in deviations from exact Euclidean invariance in the continuum approximation to this full underlying theory.

If a discretisation that respects the invariance is desired, there must be no preferred directions and this can be achieved by taking a random distribution of atoms, in other words atomic positions chosen according to a Poisson distribution so that the expected number of atoms in a given region is the mass in that region (in atomic mass units). This will produce, with high probability, an atomic configuration that does not distinguish any direction.

Having placed the atoms down, we kick away the prop of the continuum description and ask if the distribution of atoms itself could be the underlying reality; in particular how do we start with it and recover, approximately, a continuum description? To answer this question, we can use the discretisation in reverse: a continuum mass density is a good approximation to an atomic state if that atomic state could be a discretisation of the mass density. In the case of the atomic states arising from random discretisations, this is modified to: a mass density is a good approximation if the atomic state could have arisen with relatively high probability from amongst the possible discretisations. Then it must be checked that if two continua are good approximations to the same atomic state, they must be close to each other - this 
is crucial if this relationship between discrete states and continuum states is to be consistent.

Finally, we propose the atomic state as the underlying reality, reinterpret mass as a measure of the number of atoms in a region, ask whether there are atomic states which have no continuum approximation and what their meaning is, start working on a dynamics for the atoms etc.

\subsection{Discrete spacetime}

These steps are straightforward in this simple case, and can be taken in analogous fashion in the discretisation of a continuum spacetime given by a Lorentzian metric tensor on a differentiable manifold. Let us tread the same path.

What plays the role of the mass density? A good candidate for the measure of the sheer quantity of spacetime in a region is the volume of that region. It is calculated by integrating over the region the volume density given by the square root of minus the determinant of the spacetime metric, $\int_{\text {region }} \sqrt{-g} d^{4} x$ and is a covariant quantity: it is independent of the coordinates used to calculate it.

In the case of quantum gravity we have independent evidence, from the entropy of black holes for example, 9] that the scale of the discreteness is of the order of the Planck scale formed from the three fundamental constants, $G, \hbar$ and $c$. If the Planck length is defined to be $l_{p}=\sqrt{\kappa \hbar}$ where $\kappa=8 \pi G$ and we have set $c=1$, then the fundamental unit of volume will be $V_{f} \equiv \nu l_{p}^{4}$ where $\nu$ is a number of order one and yet to be determined. In order to discretise a spacetime, we distribute the atoms of spacetime, which we will call simply "elements", in the spacetime in such a way that the number of them in any sufficiently large region is approximately the volume of that region in fundamental volume units.

Analogous to the question of Euclidean invariance in the material example, we must ask: do we want to preserve Lorentz invariance or not? We might think of laying down a grid of coordinates on spacetime marked off at $\nu^{1 / 4} l_{p}$ intervals and placing an element at every grid vertex. The problem that immediately arises is that it is not a covariant prescription and this manifests itself dramatically in frames highly boosted with respect to the frame defined by the coordinates, where the distribution of elements will not be uniform but will contain large void regions in which there are no elements at all (see e.g. reference [10] for a picture). Such a coordinate-dependent discretisation will therefore violate Lorentz invariance. This is a much more serious matter than the breaking of Euclidean invariance for a coordinate-based discretisation of the material. The breaking of Lorentz invariance manifests itself by a failure of the distribution of elements to be uniform at all in highly boosted frames. If such a discrete entity were to be proposed as fundamental, it would have to be concluded that in highly boosted frames there can be no manifold description at all.

There is as yet no evidence that Lorentz invariance is violated so let us try to maintain it in quantum gravity. In seeking a Lorentz invariant discretisation process the crucial insight is, again, that the discretisation should be random. [5] It 
is performed via a process of "sprinkling" which is the Poisson process of choosing countable subsets of the spacetime for which the expected number of points chosen from any given region of spacetime is equal to its volume in fundamental units. That this process is exactly Lorentz invariant in Minkowski spacetime is a consequence of the fact that the Minkowskian volume element is equal to the Euclidean volume element on $\mathbb{R}^{n}$ and the fact that the Poisson process in $\mathbb{R}^{n}$ is invariant under any volume preserving map (see e.g. reference [11]).

The sprinkling process results in a uniform distribution of elements in the spacetime but this set of discrete elements cannot, by itself, provide a possible fundamental description of spacetime. Here, our analogy with a simple mass density in a box breaks down. When, having constructed a distribution of atoms in the box, we take away the continuum mass density, like whisking away the tablecloth from under the crockery, the atoms retain their positions in space because Euclidean space is an assumed background - the table - for the whole setup. But in the case of quantum gravity, the sprinkled elements are meant to be the spacetime and if we whisk spacetime away from the elements we have sprinkled into it - removing the table not just the tablecloth - the elements just collapse into a heap of unstructured dust. The sprinkled elements must be endowed with some extra structure.

We already know that by counting elements we can recover volume information in the continuum approximation. Powerful theorems in causal analysis [12, 13, 14] show that what is needed in the continuum to complete volume information to give the full spacetime geometry is the causal structure of a spacetime. ${ }^{1}$

The causal structure of a spacetime is the totality of the information about which events can causally influence which other events. For each point $p$ of the spacetime, we define the set $J^{-}(p)\left(J^{+}(p)\right)$ the causal past (future) of $p$, to be the set of points, $q$, in the spacetime for which there is a future (past) directed causal curve - a curve with an everywhere non-spacelike, future (past) pointing tangent vector - from $q$ to $p$. The collection of all these causal past and future sets is the causal structure of the spacetime. This is often colloquially called the "light cone structure" because the boundaries of these sets from a point $p$ are the past and future lightcones from $p$ and the sets themselves are the lightcones and their interiors.

Let us therefore, in our discretisation procedure, endow the elements sprinkled into the spacetime with the order given by the spacetime causal structure: elements $e_{i} \prec e_{j}$ if they are sprinkled at points $p_{i}$ and $p_{j}$ respectively in the continuum such that $p_{i} \in J^{-}\left(p_{j}\right)$. The set of sprinkled elements with this induced order is a causal set satisfying the axioms given above.

Now we give the causal set independence from the spacetime. To recover from it approximately the continuum we discretised, we follow the guidance of the example of the substance in a box: a spacetime $M$ is a good approximation to a causal set

\footnotetext{
${ }^{1}$ The theorems apply to spacetimes that satisfy a certain global causality condition - past and future distinguishability - which means that distinct points have distinct causal pasts and futures and which we will assume for every continuum spacetime referred to here. We can say that the theorems imply that causal set theory predicts spacetime must satisfy this condition because only such spacetimes will be able to approximate a causal set.
} 
$C$ if $C$ could have arisen from $M$ by the discretisation process we have described (sprinkling and endowing with induced order) with relatively high probability.

As mentioned in the material-in-a-box example, consistency requires that if two continua are good approximations to the same discretum, they should be close to each other. This is a central conjecture, the Hauptvermutung, of causal set theory and it is surprisingly hard even to formulate it precisely due to the difficulty of defining a notion of distance on the space of Lorentzian manifolds. We use the intuitive idea constantly - how else would it make sense to talk of one spacetime being a small perturbation of another - but only recently has progress been made in this direction. 15, 16] This progress has been inspired by causal sets, in particular by utilising and comparing the different probability distributions on the space of causal sets given by the sprinkling processes into different Lorentzian manifolds. If it is the case that using sprinklings is the only way covariantly to say what we mean by two Lorentzian spacetimes being close, it would be further evidence for causal sets as the deep structure.

\subsection{Reassessed in a quantal light}

It may be argued that the above steps leading to the proposal of causal sets for quantum gravity kinematics have been taken under the assumption that the continuum is an approximation to one single discrete spacetime whereas Quantum Mechanics would suggest that a continuum spacetime is better characterised as corresponding to a coarse grained set of many discreta. This point has validity and in addressing it, we are drawn into the realm of dynamics and the question of what form a quantum dynamics for causal sets might take, the subject of the next section. Certainly, the statement that the number and ordering of causal set elements correspond to continuum volume and causal structure, respectively, will have to be judged and interpreted in the light of the full dynamical theory. In the meantime, however, Quantum Mechanics need make us no more squeamish about the statement, "Spacetime is a causal set", than about the statement, "Things are made of atoms."

Even if it turns out that only a whole bunch of discreta can properly be said to correspond to a continuum spacetime, we can still make the claim that discrete data can give rise, in a Lorentz invariant manner, to a continuum spacetime if they are organised as a causal set. In this case, we would say that the data common to, or shared by, each member of the bunch of discreta - a coarse graining of them all - is a causet. The question would then be, what discretum can be coarse grained to give a causet?

The answer is, another "finer" causet! Indeed causets admit a notion of coarse graining that is consistent with the inverse procedures of discretisation and continuum approximation given above because it is itself a random process. To perform

a $\frac{1}{q}: 1$ coarse graining of a causet, go through it and throw away each element with fixed probability $p=1-q$. A causet $a$ is a coarse graining of a causet $b$ if $a$ could have resulted with relatively high probability from the process of coarse graining $b$. 
On this view, a spacetime $M$ could correspond dynamically to a set of "microscopic" states which are causets with no continuum approximation at all, but which have a common coarse graining to which $M$ is a good approximation.

It should be mentioned that this process of coarse graining allows the notion of scale dependent topology to be realised in causal set theory. Many quantum gravity workers have the intuitive picture that at scales close to the Planck scale, the topology of spacetime will be complicated by many wormholes and other nontrivial topological excitations, but at every day scales the topology is trivial. As attractive as this idea is, I know of no way in the continuum to make it concrete. If spacetime is a causet, however, coarse graining it at different scales (i.e. with different deletion probabilities $p$ ) gives rise to different causets which may have continuum approximations with different topologies, including different dimensions.

\section{Dynamics}

If we imagine a bag containing all the causets with $N$ elements, where $N$ is some immensely large number, then drawing out of the bag a causet uniformly at random will result, with probability approaching 1 as $N$ tends to infinity, in a causet with a very specific and rather surprising structure. This structure is of three "levels": the first level is of elements with no ancestors and has roughly $N / 4$ members, the second is of elements with ancestors in level 1 and has roughly $N / 2$ members and the remaining elements are in level 3 with ancestors in level 2. [17.

These 3-level causets have no continuum approximation - they are universes that "last" only a couple of Planck times. If the fundamental reality is a causal set then we have to explain why the causet that actually occurs is a manifold-like one and not one of the, vastly more numerous, 3-level causets. This is the causal set version of a problem that is common to all discrete approaches to quantum gravity: the sample space of discreta is always dominated in sheer numbers by the non-manifold-like ones and a uniform distribution over the sample space will render these bad ones overwhelmingly more likely. We need a dynamics to cancel out this "entropic" effect and to produce a measure over the sample space that is peaked on the manifold-like entities.

As mentioned in the introduction, the broad goal of quantum gravity includes the unification of observer with observed (or rather the elimination of the observer as fundamental). Thus, finding a dynamics for quantum gravity, involves a resolution of the vexed problem of the "Interpretation of Quantum Mechanics." There is no consensus on how this is to be achieved and this difficulty could be seen as a severe obstacle in the quest for quantum gravity. Turning the problems around, however, the requirements of quantum gravity, for example general covariance, might be taken as a guide for how to approach Quantum Mechanics in general. Indeed, quantum gravity points to an approach to Quantum Mechanics that is based on the histories of a system. 


\subsection{A histories framework for quantum causal sets}

The reader may already be aware that the terms "quantum state" and "Hilbert space" etc. have been conspicuous by their absence in the present account. I have avoided them for reasons bound up with our broad goals in quantum gravity. The standard approach to Quantum Mechanics based on quantum states, i.e. elements of a Hilbert space, is tied up with the Copenhagen interpretation with its emphasis on "observables" and "observers". The quantum state is a state "at a moment of time" (in the Schrödinger picture) and to define it requires a foliation of spacetime by spatial hypersurfaces. Taking the principle of general covariance fully to heart suggests that our goals are better served by instead maintaining the fully spacetime character of reality inherent in General Relativity, which points to the framework of the "sum-over-histories" for quantum gravity. 18, 19, 9]

The bare bones structure of a histories quantum theory is a sample space, $\Omega$ of possible histories of the system in question - for us, causal sets - and a dynamics for the system expressed in terms of a "quantum measure" $\mu$, on $\Omega$. I will say more about the quantum measure below. For now, note that were $\mu$ a probability measure, this would be the familiar case of a classical stochastic theory and indeed a histories quantum theory can be thought of as a generalisation of such a theory. That being so, we can prepare for our task of finding a quantum dynamics by studying classical stochastic causets.

\section{$3.2 \quad$ A classical warm up}

Just as a probability measure on the space of all paths on the integers - a random walk - can be given by all the "transition probabilities" from each incomplete path, $\gamma$, to $\gamma$ plus an extra step, so a measure on the space of all possible past finite ${ }^{2}$ causets can be specified by giving all the transition probabilities from each $n$-element causet, $c$, to all possible causets formed from $c$ by adding a single new element to it. The ancestors of the newly born element are chosen from the elements of $c$ according to the distribution of the transition probabilities. This is a process of stochastic "sequential growth" of a causet. 20]

Without any restrictions, the number of such "laws of growth" is so huge that the class of them is not very useful to study. Imposing on the dynamics two physically motivated conditions severely narrows down the class, however. These conditions are discrete general covariance and Bell causality. The first condition states that the probability of growing a particular finite partial causet does not depend on the order in which the elements are born. This is recognisably a "label independence" condition and analogous to the independence of the gravitational action from a choice of coordinates. The second condition is the closest possible analogue, in this setting of causet growth, of the condition that gives rise to the Bell Inequalities in an ordinary stochastic theory in a background with fixed causal structure. It is meant to imply that a birth taking place in one region of the causet cannot be influenced

\footnotetext{
${ }^{2}$ Past finite means that every element has finitely many ancestors.
} 
by any birth in a region spacelike to the first.

The solution of these two conditions is the class of "Rideout-Sorkin" models. Each model is specified by a sequence of non-negative real numbers, $t_{0}=1, t_{1}, t_{2}, \ldots$ and the transition probabilities can be expressed in terms of these "coupling constants." The models are also known as "generalised percolations" since they generalise the dynamics called "transitive percolation" in which a newly born element independently chooses each of the already existing elements to be its ancestor with probability $p$. In generalised percolation a newly born element chooses a set of elements of cardinality $k$ to be its ancestors with relative probability $t_{k} \cdot{ }^{3}$

This family of models has proved to be a fruitful test bed for various issues that will arise in the quantum theory, most notably that of general covariance.

\subsection{The problem of general covariance}

Part of the problem of general covariance is to identify exactly how the principle manifests itself in quantum gravity and how the general covariance of General Relativity arises from it. The answer will surely vary from approach to approach but some overarching comments can be made. Part of what general covariance means is that physical quantities and physical statements should be independent of arbitrary labels. It is needed as a principle when the dynamics of the system is not expressible (or not obviously expressible) directly in terms of the label invariant quantities, but can only be stated as a rule governing the labelled system. To atone for the sin of working with these meaningless labels, the principle of general covariance is invoked and physical statements must be purged of these unphysical markers.

This is a difficult thing to do even in classical General Relativity. In quantum gravity, the issue is even more fraught because it is caught up in the quantum interpretational argy bargy. But the rough form of the problem can be understood in the following way. When the dynamics is expressed in terms of labelled quantities, we can satisfy general covariance by formally "summing over all possible labellings" to form covariant classes of things (histories, operators, whatever). But in doing so, the physical meaning of those classes is lost, in other words the relation of these classes to label-independent data is obscure. Work must then be done to recover this physical meaning. As an example, consider, in flat spacetime, the set of non-abelian gauge fields which are zero in a fixed spacetime region and all fields which are gauge equivalent to them. This is a gauge invariant set of fields, but its physical meaning is obscure. Uncovering a gauge invariant characterisation of elements of the set takes work.

In causal set theory, progress has been made on this issue. 21, 22] A RideoutSorkin dynamics naturally gives a measure on the space of labelled causets, $\tilde{\Omega}-$ the labelling is the order in which the elements are born. This labelling is unphysical, the only physical structure possessed by a causet is its partial order. General covariance requires that only the covariant subsets of $\tilde{\Omega}$ have physical meaning where a covariant

\footnotetext{
${ }^{3}$ It would be more accurate to say "proto-ancestors" because the set has to be completed by adding all its own ancestors.
} 
subset is one which if it contains a labelled causet, also contains all other labellings of it. Such subsets can be identified with subsets of the unlabelled sample space $\Omega$. For each covariant, measureable set, $A$, the dynamics provides a number $\mu(A)$, the probability that the realised causet is an element of $A$. But what is a physical, i.e. label-independent characterisation of the elements of such a set $A$ ?

We now know that given almost any causet $c$ in $\Omega$ we can determine whether or not it is in $A$ by asking countable logical combinations of questions of the form, "Is the finite poset $b$ a stem ${ }^{4}$ in $c$ ?" The only case in which this is not possible is when $c$ is a so-called rogue causet for which there exists a non-isomorphic causet, $c^{\prime}$ with exactly the same stems as $c$. The set of rogues, however, has measure zero and so they almost surely do not occur and we can simply eliminate them from the sample space.

Which finite posets are stems in $c$ is manifestly label-invariant data and the result implies that asking these sorts of questions (countably combined using "not", "and" and "or") is sufficient to exhaust all that the dynamics can tell us.

This result depends crucially on the dynamics. It would not hold for other sequential growth models in which the special type of causet which threatens to spoil the result does not have measure zero. So, though we can claim to have solved the "problem of covariance" for the Rideout-Sorkin models, when we have a candidate quantum dynamics, we will have to check whether the "stem questions" are still the whole story.

\subsection{The problem of Now}

Nothing seems more fundamental to our experience of the world than that we have those experiences "in time." We feel strongly that the moment of Now is special but are also aware that it cannot be pinned down: it is elusive and constantly slips away as time flows on. As powerful as these feelings are, they seem to be contradicted by our best current theory about time, General Relativity. There is no scientific contradiction, no prediction of General Relativity that can be shown to be wrong. But, the general covariance of the theory implies that the proper way to think of spacetime is "timelessly" as a single entity, laid out once and for all like the whole reel of a movie. There's no physical place for any "Now" in spacetime and this seems at odds with our perceptions.

In the Rideout-Sorkin sequential growth models we see, if not a resolution, then at least an easing of this tension. The models are covariant, but nevertheless, the dynamics is specified as a sequential growth. An element is born, another one is born. There is growth and change. Things happen! But the general covariance means that the physical order in which they happen is a partial order, not a total order. This doesn't give any physical significance to a universal Now, but rather to events, to a Here-and-Now.

I am not claiming that this picture of accumulating events (which will have to be

\footnotetext{
${ }^{4} \mathrm{~A}$ stem of a causet is a finite subcauset which contains all its own ancestors.
} 
reassessed in the quantum theory) would explain why we experience time passing, but it is more compatible with our experience than the Block Universe view.

One might ask whether such a point of view, that events happen in a partial order, could be held within General Relativity itself. Certain consequences have to be grappled with: if one event, $x$ has occurred, then before another event, $y$ can occur to its future, infinitely many events must occur in between and this is true no matter how close in proper time $x$ and $y$ are. Perhaps it is possible to make this coherent but, to my mind, the discreteness of causal sets makes it easier to make sense of this picture of events occurring in a partial order.

Another thing left unexplained by General Relativity is the observational fact of the inexorable nature of time: it will not stop. Tomorrow will come. We have not so far encountered an "edge" in spacetime where time simply comes to an end (nor, for that matter, an edge in space). Spacetimes with boundaries, for example a finite portion of Minkowski space, are just as much solutions of the Einstein equations as those without and General Relativity cannot of itself account for the lack of boundaries and holes in spacetime. In a Rideout-Sorkin universe, one can prove that time cannot stop. A sequential growth must be run to infinity if it is to be generally covariant and Joe Henson has proved that in a Rideout-Sorkin model every element has a descendant and therefore infinitely many descendants. 23. If such a result continues to hold true in the quantum case, not only would it prove that tomorrow will always come but also would imply that in situations where we expect there to be no continuum approximation at all, such as a Big Crunch or the singularity of a black hole, the causal set will continue to grow afterwards.

The Rideout-Sorkin models give us a tantalising glimpse of the sorts of fundamental questions that may find their answers in quantum gravity when we have it.

\subsection{The quantum case}

The mathematical structure of ordinary unitary Quantum Mechanics in its sumover-histories formulation is a generalisation of a classical stochastic theory in which instead of a probability measure there is a "quantum measure" on the sample space. ${ }^{5}$ The quantum measure of a subset, $A$, of $\Omega$ is calculated in the familiar way by summing and then squaring the amplitudes of the fine grained elements of $A$. A quantum measure differs from a classical probability measure in the phenomenon of interference between histories which leads to it being non-additive. A familar example is the double slit experiment: the quantum measure of the set of histories which go through the upper slit plus the quantum measure of the set of histories which go through the lower slit is not equal to the quantum measure of the set of all histories which end up on the screen.

After the breakthrough of the Rideout-Sorkin models, it seemed that it would be relatively straightforward to generalise the derivation to obtain a quantum measure

\footnotetext{
${ }^{5}$ Indeed Quantum Mechanics is the first of an infinite series of generalisations resulting in a heirarchy of measure theories with increasingly complex patterns of "interference" between histories. 24]
} 
for causets. Roughly speaking, instead of transition probabilities there would be transition amplitudes and the quantum measure would be constructed from them via a generalisation of "sum and square" appropriate for a non-unitary dynamics. 25] General covariance and the appropriate quantum version of the Bell Causality condition could then be solved to find the form that the transition amplitudes must take. However, it is proving difficult to find the required Quantum Bell Causality condition, not least because the condition is not known even in the case of ordinary unitary Quantum Mechanics in a background with a fixed causal structure though we do now have at least a candidate for it. 26.

Even if we had in hand a covariant, causal quantum measure, there would still remain the problem of interpreting it. The interference between histories and consequent non-additive nature of the quantum measure mean that we are exploring new territory here. Reference [27] is a first attempt at a realist interpretation for quantum measure theory. It relies on the adequacy of predictions such as, "Event $X$ is very very unlikely to occur," to cover all the predictions we want to make, which should include all the predictions we can make in standard quantum mechanics with its Copenhagen Interpretation. This adequacy is explicitly denied by Kent 28 and I have tended to agree with this judgement. However, the quantum measure is the result of taking a conservative approach to Quantum Mechanics (no new dynamics) whilst making histories primary, maintaining fundamental spacetime covariance and taking a completely realist perspective. As such it deserves to be persevered with.

\section{Conclusions}

The belabouring, in section 2 , of the correspondence between the inverse processes of discretisation and continuum approximation makes manifest a certain conservatism of causal set theory: the steps are familiar, we've been down similar roads before. Moreover, spacetime, albeit discrete, is still considered to be real. There is no replacement of spacetime by a substance of a completely different ilk, such as a collection of $D 0$-branes in 11-dimensional flat spacetime 29] to choose an example at random, as the underlying ontology. The radical kinematical input is the postulate of fundamental discreteness.

However, no matter how smooth one can make such arguments for causal sets, no scientific theory can be arrived at by pure philosophical introspection. For a start, hard scientific labour is already contained in the proof of the essential result that the causal structure fixes the spacetime metric up to local volume information. This has been strengthened by results that show that topological and geometrical information can indeed be "read off" from a causet which is a sprinkling into Minkowski spacetime. More importantly, we need to do a great deal of further work. For example, within kinematics the Hauptvermutung needs to be given a formal mathematical statement and more evidence provided for it and we need to have more results on how to read off geometrical information from a causet especially in the case of curved spacetime.

In the area of dynamics the Rideout-Sorkin models, though only classically 
stochastic, are proving invaluable for exploring issues such as the problem of general covariance. It is possible that a more or less direct generalisation of the derivation of these models will lead to the desired quantum theory. Finding a quantum dynamics is the central challenge for workers in causal set theory. I have given a somewhat sketchy account of some of the conceptual hurdles that need to be overcome before this can be achieved.

One thing that has not been explored in this article is how far causal set theory has come in the area of phenomenology, in other words in the deriving of observable consequences of the theory. It will be hard for any approach to quantum gravity to come to be universally accepted without experimental and observational evidence in the form of predictions made and verified. In this regard, causal set theory already has the advantage of a long-standing prediction of the current order of magnitude of the cosmological constant, or "dark energy density" [6, 7, 9] that has apparently now been verified. The argument leading to this prediction is heuristic - it depends on certain expectations about the quantum theory - and can only be made rigorous with the advent of the full quantum causal set dynamics. However, the sheer unexpectedness of the observational result amongst the wider community of theorists some cosmologists have called it preposterous [30] - is great encouragement to investigate the arguments further. Numerical simulations of stochastic cosmologies based on the arguments bear out the conclusion that the envelope of the fluctuations of the dark energy density tends to "track" the energy density of matter 31. Improvements would include models which allow spatial inhomogeneities in the dark energy density.

Moreover, there is great promise for further phenomenological model building. The unambiguous kinematics of the causal set approach means that there is an obvious way to try to make phenomenological models: create a plausible dynamics for matter (particles or fields, say) on the background of a causal set that is well approximated by the classical spacetime that we actually observe: Minkowski spacetime or Friedmann-Robertson-Walker spacetime, depending on the physical context. The dynamics of the matter might be classical or quantum. The limitation of such model building is that it doesn't take into account the quantum dynamics of the causal set itself, nor any back-reaction, but these models could be a first step in deriving observable effects of the underlying discreteness on phenomena such as the propagation of matter over long distances. An example of exactly this form is a model of point particle motion on a causet which leads to a prediction of a Lorentz invariant diffusion in particle momentum and therefore energy. 10] A naive application to cosmic protons doesn't work as a universal acceleration mechanism that might explain high cosmic energy rays but a quantum version might do better and the idea could be applied to other particles like neutrinos.

In causal set theory, we now have the mathematical structure that Einstein lacked, giving us a framework for a fundamentally discrete theory of spacetime which does not rely on any continuum concept as an aid. How successful it will be in realising the unification that Einstein hoped for, will be for the future to decide. But let me end by musing on a unification even beyond that of quantum gravity: the unification of kinematics and dynamics. In causal set theory as currently conceived, 
the subject of the theory and the laws by which it is governed are different in kind. This is apparent in the Rideout-Sorkin models for example. The law of growth is given by a sequence of non-negative numbers. This law is not part of physical reality which is the causal set. To a materialist like myself, it would be more satisfying if the laws themselves were, somehow, physically real; then the physical universe, meaning everything that exists, would be "self-governing" and not subject to laws imposed on it from outside. Should these nebulous ideas find concrete expression it would represent perhaps the ultimate unity of physics.

\section{Acknowledgments}

I gratefully acknowledge the influence of Rafael Sorkin on this article. Anyone familiar with his work will realise that I have plagiarised his writings shamelessly. I thank him and my collaborators on causal sets, Graham Brightwell, Raquel Garcia, Joe Henson, Isabelle Herbauts and Sumati Surya for their insights and expertise.

\section{References}

[1] A. A. Robb, Geometry of Time and Space. (Cambridge University Press, Cambridge (UK), 1936). A revised version of "A theory of Time and Space" (C.U.P. 1914).

[2] J. Stachel. Einstein and the quantum: Fifty years of struggle. In ed. R. Colodny, From Quarks to Quasars, Philosophical Problems of Modern Physics, p. 379. U. Pittsburgh Press, (1986).

[3] G. 't Hooft. Quantum gravity: a fundamental problem and some radical ideas. In eds. M. Levy and S. Deser, Recent Developments in Gravitation (Proceedings of the 1978 Cargese Summer Institute). Plenum, (1979).

[4] J. Myrheim, (1978). CERN preprint TH-2538.

[5] L. Bombelli, J.-H. Lee, D. Meyer, and R. Sorkin, Space-time as a causal set, Phys. Rev. Lett. 59, 521, (1987).

[6] R. D. Sorkin. First steps with causal sets. In eds. R. Cianci, R. de Ritis, M. Francaviglia, G. Marmo, C. Rubano, and P. Scudellaro, Proceedings of the ninth Italian Conference on General Relativity and Gravitational Physics, Capri, Italy, September 1990, pp. 68-90. World Scientific, Singapore, (1991).

[7] R. D. Sorkin. Space-time and causal sets. In eds. J. C. D'Olivo, E. NahmadAchar, M. Rosenbaum, M. P. Ryan, L. F. Urrutia, and F. Zertuche, Relativity and Gravitation: Classical and Quantum, Proceedings of the SILARG VII Conference, Cocoyoc, Mexico, December 1990, pp. 150-173. World Scientific, Singapore, (1991). 
[8] R. D. Sorkin. Causal sets: Discrete gravity (notes for the valdivia summer school). In eds. A. Gomberoff and D. Marolf, Lectures on Quantum Gravity, Proceedings of the Valdivia Summer School, Valdivia, Chile, January 2002. Plenum, (2005).

[9] R. D. Sorkin, Forks in the road, on the way to quantum gravity, Int. J. Theor. Phys. 36, 2759-2781, (1997).

[10] F. Dowker, J. Henson, and R. D. Sorkin, Quantum gravity phenomenology, lorentz invariance and discreteness, Mod. Phys. Lett. A19, 1829-1840, (2004).

[11] D. Stoyan, W. Kendall, and J. Mecke, In Stochastic geometry and its applications, chapter 2. Wiley, 2 edition, (1995).

[12] S. W. Hawking, A. R. King, and P. J. McCarthy, A new topology for curved space-time which incorporates the causal, differential, and conformal structures, J. Math. Phys. 17, 174-181, (1976).

[13] D. B. Malament, The class of continuous timelike curves determines the topology of spacetime, J. Math. Phys. 18, 1399-1404, (1977).

[14] A. V. Levichev, Prescribing the conformal geometry of a lorentz manifold by means of its causal structure, Soviet Math. Dokl. 35, 452-455, (1987).

[15] L. Bombelli, Statistical lorentzian geometry and the closeness of lorentzian manifolds, J. Math. Phys. 41, 6944-6958, (2000).

[16] J. Noldus, A lorentzian lipschitz, gromov-hausdoff notion of distance, Class. Quant. Grav. 21, 839-850, (2004).

[17] D. Kleitman and B. Rothschild, Asymptotic enumeration of partial orders on a finite set, Trans. Amer. Math. Society. 205, 205-220, (1975).

[18] S. W. Hawking, Quantum gravity and path integrals, Phys. Rev. D18, 17471753, (1978).

[19] J. B. Hartle. Space-time quantum mechanics and the quantum mechanics of space-time. In eds. J. Zinn-Justin and B. Julia, Proceedings of the Les Houches Summer School on Gravitation and Quantizations, Les Houches, France, 6 Jul - 1 Aug 1992. North-Holland, (1995).

[20] D. P. Rideout and R. D. Sorkin, A classical sequential growth dynamics for causal sets, Phys. Rev. D61, 024002, (2000).

[21] G. Brightwell, H. F. Dowker, R. S. Garcia, J. Henson, and R. D. Sorkin. General covariance and the 'problem of time' in a discrete cosmology. In ed. K. Bowden, Correlations: Proceedings of the ANPA 23 conference, August 16-21, 2001, Cambridge, England, pp. 1-17. Alternative Natural Philosophy Association, (2002). 
[22] G. Brightwell, H. F. Dowker, R. S. Garcia, J. Henson, and R. D. Sorkin, 'observables' in causal set cosmology, Phys. Rev. D67, 084031, (2003).

[23] J. Henson, (2002). Unpublished result.

[24] R. D. Sorkin, Quantum mechanics as quantum measure theory, Mod. Phys. Lett. A9, 3119-3128, (1994).

[25] X. Martin, D. O'Connor, and R. D. Sorkin, The random walk in generalized quantum theory, Phys. Rev. D71, 024029, (2005).

[26] D. Craig, F. Dowker, J. Henson, S. Major, D. Rideout, and R. Sorkin. A bell inequality analog in quantum measure theory, (2005). In preparation.

[27] R. D. Sorkin. Quantum measure theory and its interpretation. In eds. D. Feng and B.-L. Hu, Quantum Classical Correspondence: Proceedings of 4 th Drexel Symposium on Quantum Nonintegrability, September 8-11 1994, Philadelphia, PA, pp. 229-251. International Press, Cambridge, Mass., (1997).

[28] A. Kent, Against many worlds interpretations, Int. J. Mod. Phys. A5, 1745, (1990).

[29] T. Banks, W. Fischler, S. H. Shenker, and L. Susskind, M theory as a matrix model: A conjecture, Phys. Rev. D55, 5112-5128, (1997).

[30] S. M. Carroll, Dark energy and the preposterous universe. (2001).

[31] M. Ahmed, S. Dodelson, P. B. Greene, and R. Sorkin, Everpresent lambda, Phys. Rev. D69, 103523, (2004). 